\title{
Using an Expert System to Recognize and Remediate Student Errors
}

\author{
Eric Bell \\ Triton College
}

Computer-based instruction systems commonly present information and then attempt to get some feedback from the student in order to engage the student in the process. Most of these feedback mechanisms involve multiple choice quizzes or entering a value. In the case of a multiple choice quiz, the student may be directed to some remediation. Having the student enter a value in response to a specific request requires more thought and effort on the student's part, but opens the door to a seemingly infinite number of wrong answers. Can these two systems be reconciled, and is there a better way to engage the student?

An expert system developed at the school can recognize a correct response as well as an incorrect response. A system for allowing the student to enter calculation in a symbolic form has also been developed to facilitate more abstract input. If an error has been made by the student, the system presents remediation topics based on the type of error. Thus specific student error types can be addressed saving time and frustration with the instruction process. This paper presents a description of the expert system and how it is implemented and integrated into an Engineering Statics course.

\section{Introduction}

The proliferation of the personal / desk-top computer has caused many in education to examine ways to use the machines to enhance education. Unfortunately there are at least two drawbacks associated with computer use as an education enhancement tool.

One is the use of computers as a remediation tool. If the student is already having difficulties, then remedial work tends to make them even more remedial because they don't have time to put into new material. Ample evidence exists to demonstrate that students who are deficient in math related skills are at a significant disadvantage compared other students. Thus, a student entering the College without at pre-calculus background may never catch up.

Efforts to use computer-aided instruction as a study aide have been laudable. Certainly the best intentioned minds have tackled the problem, but evidence indicates only marginal progress. The primary problem seems to be that students who are having difficulties with a topic easily become frustrated as a result of misconceptions of process and erroneous facts. Misconceptions of process are usually incomplete or incorrect steps related to the problem solving process.

While, as educators, we are very familiar with the problem process for different problems, it is only because we have solved several thousand problems of a particular type. As a note, it is not proposed that we turn students into problem solving robots, but after doing enough problems, we 
(humans) internalize the process we have been doing by rote so that we are able to perceive a solution simply by examining the problem. We no longer must "think" about the process.

Incomplete steps in the problem solving process must be demonstrated to the student, and then ideally, the student demonstrates the newly learned process back. New material must be absorbed, reinforced and then demonstrated by the student for learning to have occurred and verified. Additionally, students learn best during two different modes: one-on-one; and selfdiscovery.

Students at Triton College, a community college with an open-door admissions policy, often have little access to computers at home, so most work must be done at the school itself. Despite best efforts, this means that students are limited to a fixed access schedule. This implies that access time must be efficient. A second application of computer-use is extra credit. Extra creditlike assignments rarely work, because students who are capable of doing the extra credit usually don't need it. In the case of the remedial student, computer access and subsequent use can be difficult because the student is unfamiliar with more sophisticated tools.

Students who are doing reasonably well in a course may be unwilling to spend time on a system intended for remediation because they perceive they don't need it. And in fact, they may not. And a system for providing extra credit may go neglected because they do not have enough time to spend on learning a new system and perceive the additional work as unnecessary.

Thus, a system must be an integral part of the course - required usage. The best way to encourage students to participate is to award points for each module. Students rarely do homework that is not required. Small wonder when they do not perceive that the additional work will help them when they are sure they understand the concepts. But understanding a concept alone is inadequate when the student doesn't know how to integrate that into the process of solving a problem.

Many current and older systems use multiple-choice mini-quizzes to evaluate student understanding. This automatically provides some form of hint. By providing choices that may be known to be incorrect, the remainder is the correct answer by default. Additionally, the options are limited to a minimum number as well as a maximum number of options. Anyone who has created a multiple-choice test knows that one of the greatest challenges is providing plausible alternatives to the correct answer. If a multiple-choice system is good, it requires the student to compose a solution, and then carefully examine each of the alternatives for equivalence. This is similar to trying to get a solution to look like the answer in the back of the book. Useful, but often simply an exercise in mathematical manipulation.

A system also must provide help or a hint. But this must be at a price. Since one of the objectives is to demonstrate self-actualized (initiated) activities, hints and help may be penalized as an incentive for memorization and internalization.

\section{Objectives}

Based on the above discussion, the following set of objectives can be identified. 
There are three strategic goals for the system:

- Encourage student participation

- Foster student initiated responses

- Evaluate student understanding

The tactical objectives of this system are as follows:

- Present problem solution methods by example

- Identify the type of student misunderstanding when expressed

- Remediate the error based on the type of error

- Provide incentive for participation

- Provide incentive for demonstrated learning or correct behavior

\section{Description}

The system consists of the program and data-files for presenting problems. The student identifies him or herself by name or student ID number. The student then selects a problem set from which he/she selects a particular problem. The current version of the system is stand-alone PC based, so tracking student access isn't implemented yet. Future versions of the system will track student access and attempts at each problem. The current version is an initial entry into the field. The student can change the problem set at the conclusion of each problem, so students are able to move ahead in the problem sets.

The student is then presented with the primary interaction window, shown in figure 1 . This consists of the problem display, a text based narrative, navigation buttons, and formula entry controls including hint and help buttons. An audio component may be available which students can turn off or play as many times as needed. 


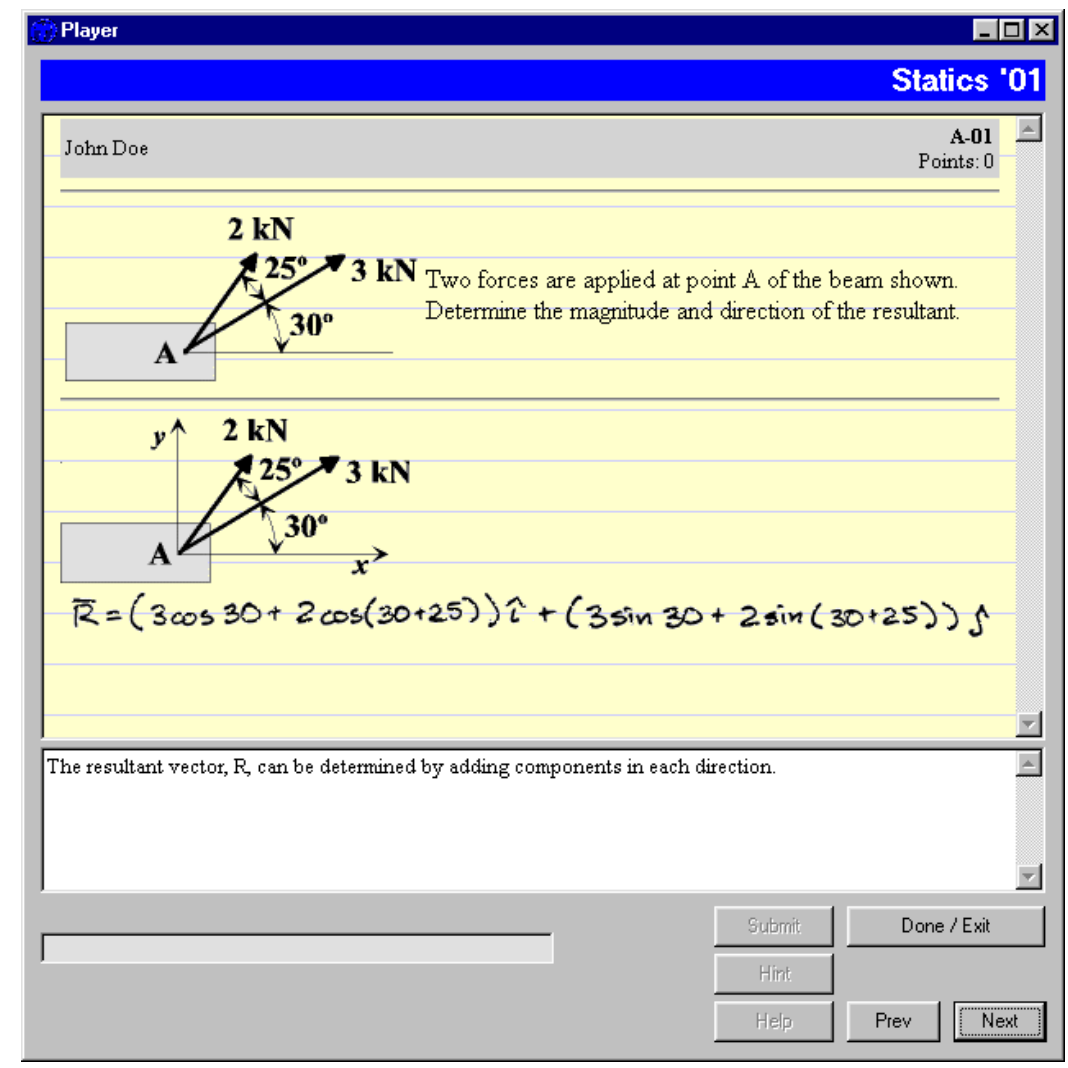

Figure 1.

At the end of the problem session, a problem summary may be printed to be turned in for credit and for the student's records. Point totals are printed on the summary along with the entire problem solving process for recording by the instructor. The summary is returned to the student in a timely manner for use as a quiz and test review tool.

As the student presses the "next step" button, each step of the problem solution is presented. The result of each step is preserved on the display. When student participation is required, the "next step" button is disabled, requiring the student to provide a response. Usually the student is required to enter an expression describing a component of a step. Where appropriate, the student may use variables as part of the symbolic expression. A list of available variables is displayed directly below the entry.

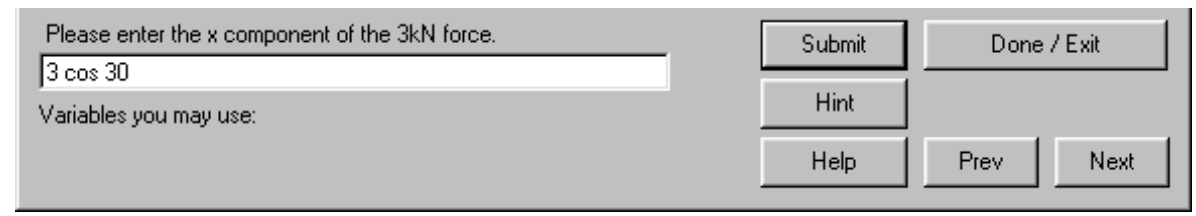

Figure 2.

As an additional feature, the expression is tested for syntactic validity whenever the student makes a change. A syntactically invalid expression is displayed in red text and disables the "submit" button. A syntactically valid expression displays the expression in black and enables the "submit" button. Thus, the student is unable to submit a syntactically invalid expression. An 
expression may be entered in any way that results in a numerically equivalent result. Thus, a student who uses $\sin \left(30^{\circ}\right)$ instead of $\cos \left(60^{\circ}\right)$ is equally correct. While pedagogically less than ideal, some compromises had to be made during implementation.

The student's response is evaluated for correctness when he/she presses or clicks the "submit" button. Simply put, the student's expression is compared with a known "correct" answer. If they match, the student is awarded full points for the step, and the student progresses to the next step.

If, on the other hand, the student's expression doesn't match the correct "answer", a search is made of a list of known erroneous answers. This is where the "expert system" comes in. If a match is found, the student is presented with that fact, along with an explanation of the type of error. A penalty may be assessed for an incorrect response. Again, because this is an expert system, a penalty value has already been associated.

A penalty need not be assessed. In the case of a student entering 3000 instead of 3 when using kilonewtons, a simple warning may suffice with no penalty. If the student's expression doesn't match any previously known error, the student is informed that the system doesn't know what they did, but to try again. No penalty is assessed, but no points are awarded either. The student cannot progress.

A hint is usually available providing the remedial or uncertain student a helpful reminder of what he/she should be looking at in the diagrams. Again, there may be a penalty, so that the student is encouraged to figure it out rather than always rely on the hint.

The hint method allows students uncertain of their skills to make some progress, rather than no progress because of misconception.

Help is also available in one of several forms. The most specific form of help provides a separate window demonstrating or showing what the student needs to solve this particular step in the problem, similar to that shown in figure 3 . The help may be as specific as obtaining the $y$ component of a vector directed toward the third quadrant as shown below. 


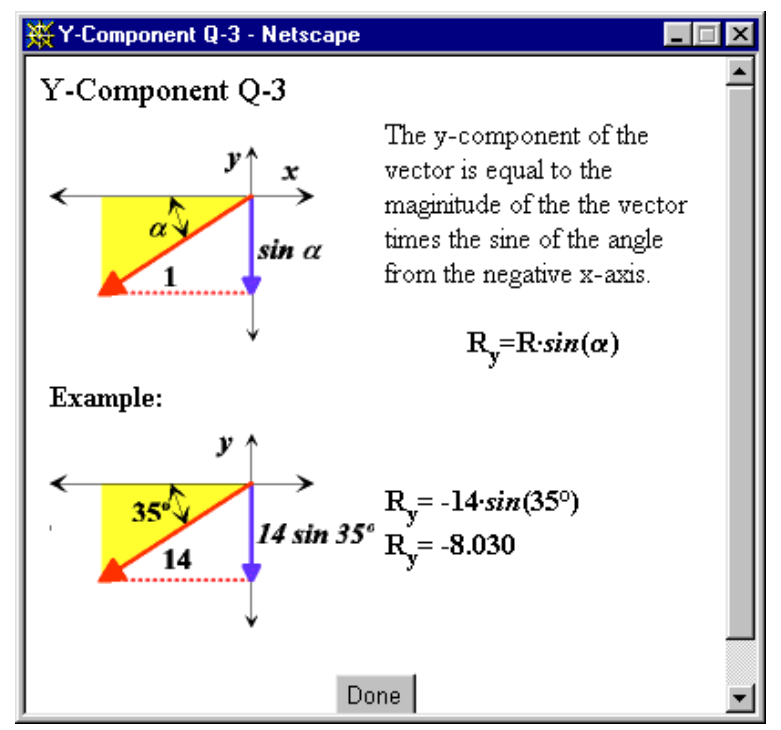

Figure 3.

As with the hints, a penalty may be assessed, encouraging students to figure it out themselves without assistance. Help can also take the form of a presentation of the topic notes, which provide a more theoretical view, but often have a simple example or two. In no case does a set of penalties result in a negative score.

\section{Pedigogy}

The system is counted as part of the homework grade. There is conventional homework assigned too. It is the author's conviction that conventional homework requires the student to originate and complete the problem solving process. The resulting homework is demonstration of this capability.

As with any homework, there may be "shared" work. While this is discouraged, it is difficult to strictly enforce. On the other hand, homework is usually performed with any number of additional resources (usually printed) as an immediate aid by example. And, as with any class, failure to perform and understand the activities demonstrated by the homework become evident in quizzes and tests.

There are often cases where the student has decided to put less than his/her full and valid effort into the learning process. This usually means incomplete or copied homework. When quizzes are administered often, the student may be caused to realize the error of his/her ways. Thus, they have an opportunity to correct it within a reasonable amount of time and without total failure.

The system also affords one-on-one interaction with the system, providing only as much help as the student requires or desires. While not providing the same emotional reinforcement as a human tutor, it has a longer attention span and does not become impatient. 


\section{Implementation}

The system has been implemented as an integral part of the Engineering Statics course. This course was selected because it requires the application of mathematics to the solution of engineering problems. And students are usually much weaker in mathematic at the beginning of the course because they have not yet seen the connection between mathematics and real problem solving. Thus, we find ourselves having to teach remedial mathematics as well as the solution process.

An authoring program has been developed that provides a more convenient means for developing and editing problems within a problem set. This also means that problem sets can be updated and downloaded while the course is in progress. This is actively discouraged, as it tends to confuse and incite students if they perceive the system to be unstable.

\section{Discussion}

One of the strategic goals of the system is to foster a response by the student that is well considered. This means that the student has to compose a solution, not just select from a menu of options. This is contrary to use of the multiple-choice quiz. The effect is that the student who knows how to solve this part of the problem simply provides the solution. And if correct, is immediately rewarded and allowed to continue.

It is also asserted that the student who has become accustomed to composing his/her own expressions is better prepared to do so in conventional homework, quizzes and tests. A study of this effect is underway. The fact that the composition is immediately evaluated and, if necessary, remediated reinforces correct and valid solutions.

It should be emphasized that this system is intended as a learning tool. It is student centered, providing the student with participation and interaction based on specific student actions. It would probably not be appropriate to use this system in a normal classroom presentation. Thus, the system is really a learning tool, not a teaching tool.

\section{Conclusion}

An automated system for delivery and grading of homework and student progress is still over the horizon. With this system, though, we are able to see that ideal system more clearly as well as make useful progress in the ideal system's desired objectives. The lessons learned from the Statics course will be applied to future versions of this course, and implemented in other courses such as Dynamics and Mechanics of Materials.

\section{ERIC BELL}

Eric Bell is currently a full time instructor at Triton College. He received his B.S. and M.S. in Mechanical Engineering from the University of Illinois of Chicago. 